\title{
Lack of association of the HMGA1 IVS5-13insC variant with type 2 diabetes in an ethnically diverse hypertensive case control cohort
}

Jason H Karnes ${ }^{1,2}$, Taimour Y Langaee ${ }^{2}$, Caitrin W McDonough ${ }^{2}$, Shin-Wen Chang ${ }^{2}$, Miguel Ramos², James R Catlin $\mathrm{Jr}^{2}$, Octavio E Casanova ${ }^{2}$, Yan Gong ${ }^{2}$, Carl J Pepine ${ }^{3}$, Julie A Johnson ${ }^{2,3}$ and Rhonda M Cooper-DeHoff ${ }^{2,3^{*}}$

\begin{abstract}
Background: Recently, the high-mobility group A1 gene (HMGA1) variant IVS5-13insC has been associated with type 2 diabetes, but reported associations are inconsistent and data are lacking in Hispanic and African American populations. We sought to investigate the HMGA1-diabetes association and to characterize IVS5-13insC allele frequencies and linkage disequilibrium (LD) in 3,070 Caucasian, Hispanic, and African American patients from the INternational VErapamil SR-Trandolapril STudy (INVEST).
\end{abstract}

Methods: INVEST was a randomized, multicenter trial comparing two antihypertensive treatment strategies in an ethnically diverse cohort of hypertensive, coronary artery disease patients. Controls, who were diabetes-free throughout the study, and type 2 diabetes cases, either prevalent or incident, were genotyped for IVS5-13insC using Taqman ${ }^{\circledR}$, confirmed with Pyrosequencing and Sanger sequencing. For LD analysis, genotyping for eight additional HMGA1 single nucleotide polymorphisms (SNPs) was performed using the Illumina ${ }^{\circledR}$ HumanCVD BeadChip. We used logistic regression to test association of the HMGA1 IVS5-13insC and diabetes, adjusted for age, gender, body mass index, and percentage European, African, and Native American ancestry.

Results: We observed IVS5-13insC minor allele frequencies consistent with previous literature in Caucasians and African Americans (0.03 in cases and 0.04 in controls for both race/ethnic groups), and higher frequencies in Hispanics ( 0.07 in cases and 0.07 in controls). The IVS5-13insC was not associated with type 2 diabetes overall (odds ratio 0.98 [0.76-1.26], $p=0.88$ ) or in any race/ethnic group. Pairwise LD $\left(r^{2}\right)$ of IVS5-13insC and rs9394200, a SNP previously used as a tag SNP for IVS5-13insC, was low $\left(r^{2}=0.47\right.$ in Caucasians, $r^{2}=0.25$ in Hispanics, and $r^{2}=0.06$ in African Americans). Furthermore, in silico analysis suggested a lack of functional consequences for the IVS5-13insC variant.

Conclusions: Our results suggest that IVS5-13insC is not a functional variant and not associated with type 2 diabetes in an ethnically diverse, hypertensive, coronary artery disease population. Larger, more adequately powered studies need to be performed to confirm our findings.

Trial registration: clinicaltrials.gov (NCT00133692)

Keywords: HMGA1, Type 2 diabetes, Genetics

\footnotetext{
*Correspondence: dehoff@cop.ufl.edu

${ }^{2}$ Department of Pharmacotherapy and Translational Research, University of

Florida, HSC PO Box 100486, Gainesville, FL 32610-0486, USA

${ }^{3}$ Division of Cardiovascular Medicine, University of Florida, PO Box 100277,

Gainesville, FL 32610-0486, USA

Full list of author information is available at the end of the article
} 


\section{Background}

Type 2 diabetes constitutes a major and growing health problem worldwide and is predicted to afflict 490 million by 2030 [1]. Type 2 diabetes has strong genetic influences and many polymorphisms have now been reproducibly associated with type 2 diabetes [2,3]. However, genome wide association studies (GWAS) explain only $10-15 \%$ of heritability and have not consistently improved diabetes risk prediction [4]. Low frequency variation may account for much of the missing heritability in type 2 diabetes risk and may help translate genetic association study results into clinical type 2 diabetes risk prediction.

Recently, the low frequency insertion polymorphism IVS5-13insC (c.136-14_136-13insC) in the high-mobility group A1 gene (HMGA1), a transcriptional regulator of the insulin receptor gene (INSR), was identified and associated with type 2 diabetes [5]. Whereas two studies have observed a significant positive association between IVS513insC and type 2 diabetes in Caucasian and Chinese populations [5,6], another study in Caucasians observed no association [7]. Data are lacking for IVS5-13insC in populations with African and Hispanic descent, which have disproportionately high type 2 diabetes prevalence [8]. Conflicting results for an HMGA1 association with type 2 diabetes and the lack of data in diverse race/ethnic groups make clinical translation of the HMGA1 IVS513insC genotyping especially difficult.

Evidence for the functional impact of the HMGA1 IVS513 ins $C$ variant is also conflicting. One study observed that HMGA1 and INSR expression was decreased in diabetic carriers of IVS5-13insC versus wild type diabetic and nondiabetic patients [5]. Additionally, INSR protein expression and insulin-binding capacity was restored in lymphoblasts obtained from diabetic IVS5-13insC carriers by HMGA1 DNA transfection. Another study observed no effect of IVS5-13insC on HMGA1 or INSR expression in adipose tissue of normoglycemic patients [7]. IVS5-13insC occurs at position -13 of HMGA1 exon 6, but the direct mechanism of the variant's effects on mRNA expression or amino acid sequence remains unclear.

We tested the association of HMGA1 IVS5-13insC with type 2 diabetes in an ethnically diverse population from the INternational VErapamil SR-Trandolapril STudy (INVEST). INVEST compared CV outcomes and NOD in hypertensive coronary artery disease patients treated with two antihypertensive treatment strategies. We also determined minor allele frequencies (MAF) and linkage disequilibrium (LD) for $H M G A 1$ variants and tested the functional impact of IVS5-13insC in silico.

\section{Methods}

\section{Study design and participants}

INVEST compared CV outcomes and incident diabetes in hypertensive, coronary artery disease patients at least
50 years of age during randomized treatment with either an atenolol-based or a verapamil sustained release (SR)based antihypertensive treatment strategy. The design, primary outcome, and NOD results have been previously published in detail [9-12]. Briefly, the verapamil SR strategy consisted of stepped therapy with verapamil SR, trandolapril add-on, dose titration, then HCTZ add-on treatment for BP control and end organ protection as necessary. The atenolol-based strategy consisted of atenolol, HCTZ add-on, dose titration, then trandolapril add-on treatment as necessary. The INVEST GENEtic Substudy (INVEST-GENES) collected DNA samples from 5,979 INVEST patients at 187 sites in the United States and Puerto Rico.

We conducted a nested case control study including cases with type 2 diabetes at baseline (prevalent diabetes) or type 2 diabetes that developed during a mean 2.8 years follow-up (incident diabetes). Type 2 diabetes was determined by patient report and by site investigators from a review of all available patient data, including use of diabetic medication and lab measures [11]. We identified age, gender, and race/ethnicity-matched controls who remained diabetes-free over a mean 2.8 years follow-up. Age matching was performed after stratification by decade and we attempted to match cases and controls in a 1:1 ratio. The institutional review boards of participating study centers approved the study protocol and all patients provided written informed consent for participation in INVEST and additional written informed consent for genetic studies. INVEST is registered at clinicaltrials.gov (NCT00133692).

\section{Genotyping}

Genotyping for HMGA1 IVS5-13insC was performed using TaqMan ${ }^{\circledR}$ (Applied Biosystems, Foster City, CA, USA) with PCR primers and probe for IVS5-13insC (PN4331349) purchased from Applied Biosystems. For IVS5-13insC genotyping quality control, 5\% of samples were genotyped in duplicate on Taqman ${ }^{\circledR}$. A total of 612 Taqman genotypes were confirmed using pyrosequencing (Biotage AB, Uppsala, Sweden), using the following PCR and sequencing primers respectively: forward-biotinylated-5'-GGGGTGGAAACAGGTGATG-3', reverse-5' -C ACTTCGCTGGGCTCCTT-3', and reverse-5' -TTCTGT AAAGACAGAGG-3'. Sanger sequencing was used to genotype 58 samples that showed discrepancies between Taqman $^{\circledR}$ and Pyrosequencing platforms.

Genotyping for eight additional HMGA1 single nucleotide polymorphisms (SNPs) was performed using the HumanCVD BeadChip and Infinium II Assay (Illumina, San Diego, CA) on 1,489 INVEST patients to perform LD analyses. The HumanCVD BeadChip contains approximately 50,000 cosmopolitan tag SNPs for 2,100 CV and metabolic-related genes [13]. HumanCVD BeadChip 
data quality was ensured in PLINK using genotype and sample call rates, concordance rates for blind duplicates, gender confirmation, cryptic relatedness using pairwise identity-by-descent, and estimation of heterozygosity using the inbreeding coefficient F [14]. Individuals were excluded if call rates were below 90 percent and SNPs were excluded if call rates were below 95 percent. In addition, 87 ancestry informative markers were genotyped in 2,860 INVEST patients to estimate Caucasian, African, and Native American ancestry using STRUCTURE [15]. Race/ethnic groups were determined by patient self-report with interaction by the study investigator [16] and confirmed using principal components analysis generated from LD-pruned HumanCVD BeadChip data and ancestry informative markers.

\section{Statistical analysis}

All statistical analyses were performed using SAS version 9.2 (SAS, Cary, NC). Differences in patient characteristics comparing cases and controls at baseline were determined using $\mathrm{t}$-tests and chi square tests, as appropriate. Deviations from Hardy Weinberg Equilibrium were assessed using a chi square test. Multi-variable logistic regressions were performed overall and by race/ethnic group to determine odds ratios (ORs) and 95\% confidence intervals $(95 \% \mathrm{CIs})$ for type 2 diabetes in IVS5$13 i n s C$ variant carriers versus non-variant carriers. Variables for adjustment included age, gender, and body mass index (BMI) in order to maintain consistency with previously published HMGA1 analyses [5,7]. Percentage of Caucasian, African, and Native American ancestry, as estimated by ancestry informative markers, was included as a variable for adjustment in analyses where all race/ ethnic groups were combined.

The combined race/ethnic group analysis was considered primary with alpha $=0.05$, since IVS5- 13 ins $C$ was presumed to be a functional SNP with similar consequences across race/ethnic groups, based on previously published reports [5,7]. Assuming a MAF of 0.05 and OR of 1.40 , we had $84 \%$ power to detect an association between the IVS5-13insC variant and type 2 diabetes in a dominant model in the overall population. Assuming a MAF of 0.05 and OR of 1.40, we had 61\% power in Hispanics, $44 \%$ power in whites, and $18 \%$ power in blacks to detect an association by race/ethnicity. Pairwise LD values $\left(\mathrm{r}^{2}\right)$ and LD plots were generated by race/ethnic group using Haploview [17]. We predicted in silico functional consequences of the IVS5-13insC variant using SNPNexus [18] and ESEfinder 3.0 [19].

\section{Results}

We identified 446 incident type 2 diabetes cases over a mean 2.8 years of follow-up and genotyped an additional 1329 prevalent type 2 diabetes cases in INVEST-GENES.
At baseline, patients with incident or prevalent type 2 diabetes had higher BMI and lower diastolic BP versus age, race/ethnicity, and gender-matched controls (Table 1). Cases also had a higher prevalence of hypercholesterolemia, left ventricular hypertrophy, and congestive heart failure.

The IVS5-13insC variant did not deviate from HWE in any race/ethnic group (Table 2). All HMGA1 SNPs had call rates above 95 percent and no SNPs failed quality control based on call rate, Hardy Weinberg equilibrium testing, concordance with blind duplicates, or estimation of heterozygosity using the inbreeding coefficient $\mathrm{F}$. The genomic inflation factor lambda was 1.03 for INVEST individuals genotyped for the HMGA1 IVS5-13insC variant, suggesting minimal population stratification in genotyped individuals. For the IVS5-13insC variant, concordance of duplicates on Taqman ${ }^{\circledR}$ was $99 \%$ and concordance between Pyrosequencing and Taqman ${ }^{\circledR}$ was $95 \%$. In Caucasians, the MAF of IVS5-13insC was 0.03 in diabetic cases and 0.04 in controls. The frequency of IVS5-13insC was similar in African American individuals (0.03 in cases and 0.04 in controls) and highest in Hispanics (0.07 in cases and 0.07 in controls). No significant associations were observed between the IVS5-13insC variant and diabetes overall (OR 0.98 95\%CI 0.76-1.26, $\mathrm{p}=0.88$ ). The IVS513insC variant was not associated with diabetes in any race/ethnic group (Table 2).

Pairwise LD of HMGA1 SNPs by race/ethnicity is presented in Figure 1. Pairwise LD $\left(\mathrm{r}^{2}\right)$ of IVS5-13insC and rs9394200, the SNP previously used as a tag SNP for IVS5-13insC [7], was 0.47 in Caucasians, 0.25 in Hispanics, 0.06 in African Americans. In silico functional analysis of IVS5-13insC revealed no predicted changes to amino acid sequence, conserved transcription factor binding sites, CpG islands, or miRNA regulatory sites. IVS5-13insC was not predicted to cause the creation or disruption of splice sites. However, in silico evaluation of exonic splice enhancer (ESE) sites revealed that the $C$ insertion of IVS5-13insC creates an additional ESE motif for SF2/ASF (IgM-BRCA1).

\section{Discussion}

In the present study, we observed that the HMGA1 IVS5-13insC variant was not associated with diabetes in the INVEST-GENES population overall or in any race/ ethnic group. We observed a frequency of IVS5-13insC consistent with previously published studies in Caucasians $[5,7]$ and observed an increased frequency of this variant in Hispanics. Furthermore, pairwise LD in our population suggests that IVS5-13insC is not effectively tagged by rs9394200 in any race/ethnic group and in silico analysis revealed minimal evidence of putative functional consequences of the IVS5-13insC variant.

Our observations extend existing knowledge of genetic determinants of type 2 diabetes and are consistent with 
Table 1 Characteristics of type 2 diabetes cases and controls at baseline

\begin{tabular}{|c|c|c|c|}
\hline Characteristic* & Type 2 diabetes cases $(n=1775)$ & Controls $(n=1295)$ & $p$ value $^{\dagger}$ \\
\hline Age (years) & $65.8(9.2)$ & $65.7(9.1)$ & 0.93 \\
\hline Female, n (\%) & $1,014(57)$ & $731(56)$ & 0.71 \\
\hline $\mathrm{BMI}\left(\mathrm{kg} / \mathrm{m}^{2}\right)$ & $30.6(5.6)$ & $28.9(5.4)$ & $<0.0001$ \\
\hline Race/ethnicity, n (\%) & & & 0.15 \\
\hline Caucasian & $608(34)$ & $493(38)$ & \\
\hline Hispanic & $937(53)$ & $642(50)$ & \\
\hline African American & $216(12)$ & $153(12)$ & \\
\hline \multicolumn{4}{|l|}{ Blood pressure (mm Hg) } \\
\hline Systolic & $149(19)$ & $148(18)$ & 0.32 \\
\hline Diastolic & $85(11)$ & $86(10)$ & 0.0008 \\
\hline Hypercholesterolemia, n (\%) ${ }^{\ddagger}$ & $1,017(57)$ & $673(52)$ & 0.003 \\
\hline History of LVH, n (\%) & $319(18)$ & $174(13)$ & 0.0007 \\
\hline History of $\mathrm{CHF}, \mathrm{n}(\%)^{* *}$ & $84(5)$ & $25(2)$ & $<0.0001$ \\
\hline History of smoking, n (\%) & $706(40)$ & $507(39)$ & 0.73 \\
\hline
\end{tabular}

BMI indicates body mass index; LVH, left ventricular hypertrophy; CHF, congestive heart failure.

*Values are mean \pm standard deviation unless otherwise noted. $† P$ values represent t-tests and chi square tests where appropriate. $\neq$ History of or currently taking lipid-lowering medications. **New York Heart Association Class I-III.

the findings of Marquez et al., which suggest a lack of association between IVS5-13insC and type 2 diabetes [7]. Marquez et al. also found that the IVS5-13insC variant is not functional, which is supported by our lack of observed association in multiple race/ethnic groups and our in silico functional results which predicted no important consequences from this variability. Our findings are inconsistent with two studies that observed a significant association between IVS5-13insC and type 2 diabetes $[5,6]$. This discrepancy may be due to differences in race/ethnicity of our population compared with other studies. However, a functional IVS5-13insC variant would be expected to have similar effects across multiple race/ethnicity groups.

Although our association analysis is consistent with the findings of Marquez et al. [7], our observation of low LD between IVS5-13insC and rs9394200 in all race/ ethnic groups suggests that rs9394200 is not an appropriate tag SNP for IVS5-13insC. In Hapmap, the MAF of the $\mathrm{T}$ allele for rs9394200 is similar to the MAF for IVS5-13insC in Caucasians (0.03), but is 0.45 in Yorubans, further suggesting that rs9394200 does not adequately tag IVS5-13insC, especially in non-Caucasian populations. Furthermore, rs9394200 is 5000 base pairs downstream from the of HMGA1 $3^{\prime}$ end and is represented on arrays utilized by type 2 diabetes GWAS $[3,20]$. Therefore, if rs9394200 were a significant contributor to risk for diabetes, it likely would have been identified in the GWAS analyses.

Our observation of a lack of association in Hispanics and African Americans suggests that IVS5-13insC may be associated with diabetes only in Caucasian individuals. While we did not observe even a trend towards association in our Caucasian population, we acknowledge that

Table 2 IVS5-13insC genotype frequencies and associations with diabetes overall and by race/ethnicity

\begin{tabular}{|c|c|c|c|c|c|c|c|}
\hline & & & & Unadjusted & & Adjusted & \\
\hline Race/ethnic group & MAF (cases) & MAF (controls) & HWE $p$ value* & Odds ratio $(95 \% \mathrm{Cl})$ & $p$ value & Odds ratio $(95 \% \mathrm{Cl})^{\dagger}$ & p value ${ }^{\dagger}$ \\
\hline Overall & 0.053 & 0.052 & - & $1.00(0.79-1.26)$ & 0.98 & $0.98(0.76-1.26)$ & 0.88 \\
\hline \multicolumn{8}{|l|}{$(n=3070)$} \\
\hline Caucasian & 0.028 & 0.036 & 0.40 & $0.75(0.46-1.22)$ & 0.25 & $0.95(0.44-2.06)$ & 0.90 \\
\hline \multicolumn{8}{|l|}{$(n=1101)$} \\
\hline Hispanic & 0.074 & 0.070 & 0.20 & $1.09(0.82-1.45)$ & 0.57 & $0.79(0.49-1.25)$ & 0.31 \\
\hline \multicolumn{8}{|l|}{$(n=1579)$} \\
\hline African American $(n=369)$ & 0.030 & 0.039 & 0.10 & $0.83(0.36-1.90)$ & 0.65 & $1.51(0.48-4.74)$ & 0.48 \\
\hline
\end{tabular}

95\% Cl indicates 95\% confidence interval; MAF, minor allele frequency, HWE, Hardy Weinberg Equilibrium.

*Hardy Weinberg Equilibrium p value calculated using a chi square test by race/ethnicity in non-diabetic patients. $\dagger$ Generated using logistic regression in a dominant model adjusted for age, gender, body mass index, and Caucasian, African, and Native American ancestry as estimated by ancestry informative markers. 


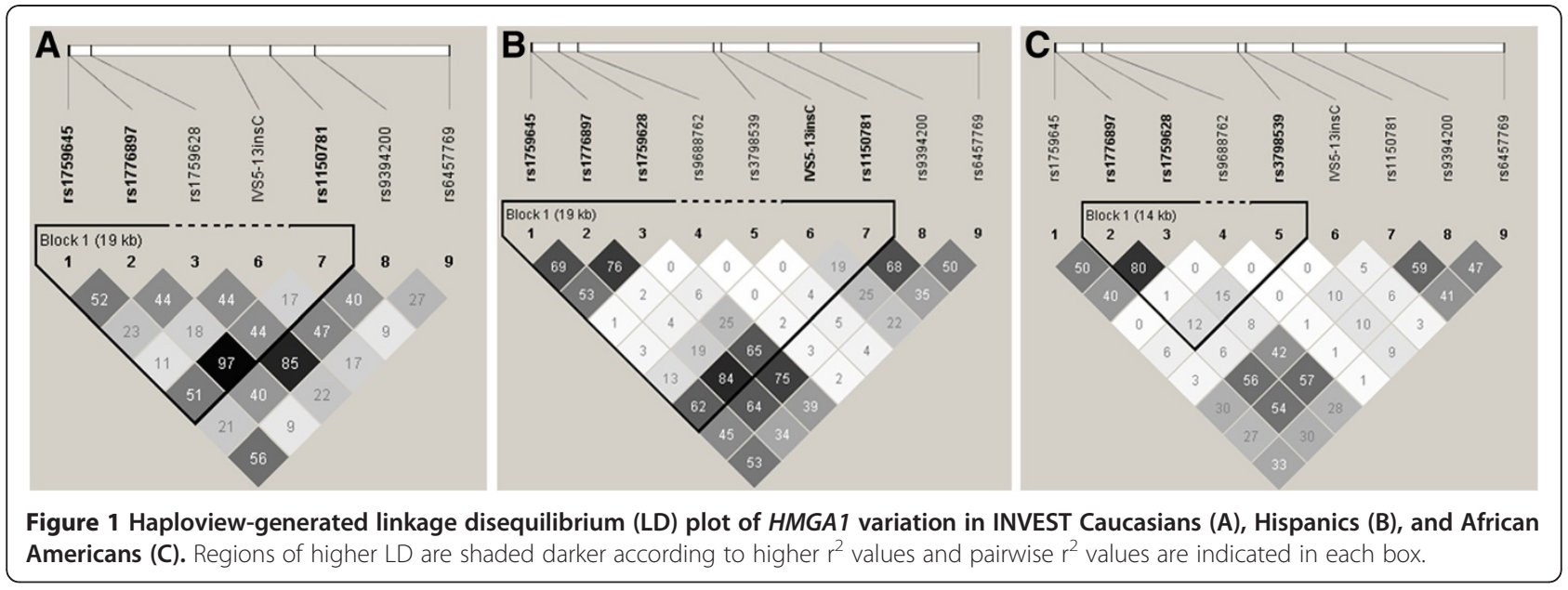

our power to observe an association in Caucasians is lower than in the previously published studies. Although we had 84 percent power to detect an association in our overall population, we had inadequate power to definitively conclude an association in by race/ethnicity analyses.

Our in silico analysis did not reveal any direct mechanism of the IVS5-13insC variant's effects on mRNA expression or amino acid sequence, suggesting that the variant is not likely to be functional. Although the results of Chiefari et al. suggested a functional effect of the IVS5-13insC variant on HMGA1 and INSR mRNA and protein expression in monocytes [5], Marquez et al. found no effect of the variant on mRNA expression in adipose tissue. The differences in observed effect on mRNA expression may potentially be explained by differential effects of the variant on transcription in monocytes and adipose tissue. The apparent functional effect of IVS5-13insC observed by Chiefari et al. may also be confounded by the lack of evaluation of IVS5-13insC variant carriers versus non-carriers among non-diabetic controls [7] or potential treatment with glucose-lowering medications in diabetic patients from whom monocytes were collected [21]. Finally, IVS513insC may have an unknown functional mechanism not identified by in silico tools used in this study. Our study has several limitations worthy of mention. We recognize the potential for false negative results in our analyses, especially in African Americans, considering the low frequency of the variant and the limited power to detect associations within each race/ethic group. Our observations require replication in independent populations of similar race/ethnic makeup. In INVEST, incident and prevalent diabetes diagnosis was based on investigator reports, but the diabetes phenotype is well described in a previous publication [11], the accuracy of such reporting has been verified by others [22], and has been used in other trials [23-25]. INVEST investigator-reported diabetes phenotypes have also been used in a large scale gene-centric meta-analysis with HumanCVD BeadChip data [26], suggesting validity with regard to the diabetes phenotype and genetic association analysis. Although our HMGA1 association analysis may be confounded if control patients eventually develop diabetes after study follow-up, the high mean age and lower mean BMI suggests that our control patients are less likely to develop type 2 diabetes. In addition, although concordance within the Taqman ${ }^{\circledR}$ genotyping platform was high, our concordance between Taqman ${ }^{\circledR}$ and Pyrosequencing was $95 \%$, suggesting some disagreement between platforms. However, Hardy Weinberg tests did not indicate genotype error and genotype error was minimized by utilization of Sanger sequencing for discrepancy confirmation.

\section{Conclusions}

Our results suggest that the HMGA1 IVS5-13insC is not associated with type 2 diabetes and may not have an important functional role in diabetes pathogenesis. We also provide frequency and LD data for Hispanic and African American populations, which have higher prevalence of type 2 diabetes. Our results also suggest that rs9394200 is not an effective tag SNP for HMGA1 IVS5-13insC, especially in non-Caucasian populations. Functional studies and replication of these associations are needed to better define the potential role of $H M G A 1$ variants in predicting type 2 diabetes development. Although the current study suggests the lack of a functional role for IVS5-13insC, further study of HMGA1 is warranted to clarify the role of this gene in diabetes pathogenesis. Larger, more adequately powered studies need to be performed to confirm our findings.

\section{Competing interests}

The authors declare that they have no competing interests.

\section{Authors' contributions}

JHK drafted the manuscript and performed statistical analysis. TYL, JRC, MR, OEC, and SWC performed genotyping and helped draft the manuscript. CWM and YG performed HumanCVD BeadChip quality control procedures, 
contributed to statistical analysis and helped draft the manuscript. CJP, JAJ, and RCD conceived of the study, participated in its design and coordination, and helped draft the manuscript. All authors read and approved the final manuscript.

\section{Acknowledgements}

This study was funded by NIH grant U01 GM074492, R01 HL74730, HL086558 (RCD), and TL1RR029888 (JHK), and grants from Abbott Laboratories and the University of Florida Opportunity Fund. We thank Ben Burkley, Lynda Stauffer, and Cheryl Galloway for processing and genotyping samples.

\section{Author details}

'Division of Clinical Pharmacology, Vanderbilt University, 1275 Medical Research Building IV, Nashville, TN 37232-0575, USA. ${ }^{2}$ Department of Pharmacotherapy and Translational Research, University of Florida, HSC PO Box 100486, Gainesville, FL 32610-0486, USA. ${ }^{3}$ Division of Cardiovascular Medicine, University of Florida, PO Box 100277, Gainesville, FL 32610-0486, USA.

Received: 5 October 2012 Accepted: 7 January 2013

Published: 9 January 2013

\section{References}

1. Zhang P, Zhang X, Brown J, Vistisen D, Sicree R, Shaw J, Nichols G: Global healthcare expenditure on diabetes for 2010 and 2030. Diabetes Res Clin Pract 2010, 87:293-301.

2. Herder C, Roden M: Genetics of type 2 diabetes: pathophysiologic and clinical relevance. Eur J Clin Invest 2011, 41:679-692.

3. Voight BF, Scott LJ, Steinthorsdottir V, Morris AP, Dina C, Welch RP, Zeggini E, Huth C, Aulchenko YS, Thorleifsson G, et al: Twelve type 2 diabetes susceptibility loci identified through large-scale association analysis. Nat Genet 2010, 42:579-589.

4. Talmud PJ, Hingorani AD, Cooper JA, Marmot MG, Brunner EJ, Kumari M, Kivimaki M, Humphries SE: Utility of genetic and non-genetic risk factors in prediction of type 2 diabetes: Whitehall II prospective cohort study. BMJ 2010, 340:b4838.

5. Chiefari E, Tanyolac S, Paonessa F, Pullinger CR, Capula C, liritano S, Mazza T, Forlin M, Fusco A, Durlach V, et al: Functional variants of the HMGA1 gene and type 2 diabetes mellitus. JAMA 2011, 305:903-912.

6. Liu L, Ding H, Wang HR, XU YJ, Cui GL, Wang PH, Yuan G, Yu XF, Wang DW: Polymorphism of HMGA1 is associated with increased risk of type 2 diabetes among Chinese individuals. Diabetologia 2012, 55:1685-1688.

7. Marquez M, Huyvaert M, Perry JR, Pearson RD, Falchi M, Morris AP, Vivequin S, Lobbens S, Yengo L, Gaget S, et al: Low-frequency variants in HMGA1 are not associated with type 2 diabetes risk. Diabetes 2012, 61:524-530.

8. Roger VL, Go AS, Lloyd-Jones DM, Benjamin EJ, Berry JD, Borden WB, Bravata DM, Dai S, Ford ES, Fox CS, et al: Executive Summary: Heart Disease and Stroke Statistics-2012 Update: A Report From the American Heart Association. Circulation 2012, 125:188-197.

9. Pepine CJ, Handberg-Thurmond E, Marks RG, Conlon M, Cooper-DeHoff R, Volkers $P$, Zellig P: Rationale and design of the International Verapamil SR/Trandolapril Study (INVEST): an Internet-based randomized trial in coronary artery disease patients with hypertension. J Am Coll Cardiol 1998, 32:1228-1237.

10. Pepine CJ, Handberg EM, Cooper-DeHoff RM, Marks RG, Kowey P, Messerli FH, Mancia G, Cangiano JL, Garcia-Barreto D, Keltai M, et al: A calcium antagonist vs a non-calcium antagonist hypertension treatment strategy for patients with coronary artery disease. The International VerapamilTrandolapril Study (INVEST): a randomized controlled trial. JAMA 2003, 290:2805-2816.

11. Cooper-Dehoff R, Cohen JD, Bakris GL, Messerli FH, Erdine S, Hewkin AC, Kupfer S, Pepine CJ: Predictors of development of diabetes mellitus in patients with coronary artery disease taking antihypertensive medications (findings from the INternational VErapamil SR-Trandolapril STudy [INVEST]). Am J Cardiol 2006, 98:890-894.

12. Cooper-DeHoff RM, Handberg EM, Mancia G, Zhou Q, Champion A, Legler UF, Pepine CJ: INVEST revisited: review of findings from the International Verapamil SR-Trandolapril Study. Expert Rev Cardiovasc Ther 2009, 7:1329-1340.

13. Keating BJ, Tischfield S, Murray SS, Bhangale T, Price TS, Glessner JT, Galver L, Barrett JC, Grant SF, Farlow DN, et al: Concept, design and implementation of a cardiovascular gene-centric $50 \mathrm{k}$ SNP array for large-scale genomic association studies. PLoS One 2008, 3:e3583.

14. Purcell S, Neale B, Todd-Brown K, Thomas L, Ferreira MA, Bender D, Maller J, Sklar P, de Bakker PI, Daly MJ, Sham PC: PLINK: a tool set for wholegenome association and population-based linkage analyses. Am J Hum Genet 2007, 81:559-575.

15. Pritchard JK, Stephens M, Donnelly P: Inference of population structure using multilocus genotype data. Genetics 2000, 155:945-959.

16. Cooper-DeHoff RM, Aranda JM Jr, Gaxiola E, Cangiano JL, Garcia-Barreto D, Conti CR, Hewkin A, Pepine CJ: Blood pressure control and cardiovascular outcomes in high-risk Hispanic patients-findings from the International Verapamil SR/Trandolapril Study (INVEST). Am Heart J 2006, 151:1072-1079.

17. Barrett JC, Fry B, Maller J, Daly MJ: Haploview: analysis and visualization of LD and haplotype maps. Bioinformatics 2005, 21:263-265.

18. Chelala C, Khan A, Lemoine NR: SNPnexus: a web database for functional annotation of newly discovered and public domain single nucleotide polymorphisms. Bioinformatics 2009, 25:655-661.

19. Cartegni L, Wang J, Zhu Z, Zhang MQ, Krainer AR: ESEfinder: A web resource to identify exonic splicing enhancers. Nucleic Acids Res 2003, 31:3568-3571.

20. Burton PR, Clayton DG, Cardon LR, Craddock N, Deloukas P, Duncanson A, Kwiatkowski DP, McCarthy Ml, Ouwehand WH, Samani NJ, et al: Genomewide association study of 14,000 cases of seven common diseases and 3,000 shared controls. Nature 2007, 447:661-678.

21. Froguel P, Marquez M, Cauchi S: Response to comment on: Marquez et al. Low-frequency variants in HMGA1 are not associated with type 2 diabetes risk. Diabetes 2012;61:524-530. Diabetes 2012, 61:e15.

22. Goldman N, Lin IF, Weinstein M, Lin YH: Evaluating the quality of selfreports of hypertension and diabetes. J Clin Epidemiol 2003, 56:148-154.

23. Aguilar D, Solomon SD, Kober L, Rouleau JL, Skali H, McMurray JJ, Francis GS, Henis M, O'Connor CM, Diaz R, et al: Newly diagnosed and previously known diabetes mellitus and 1-year outcomes of acute myocardial infarction: the VALsartan In Acute myocardial iNfarcTion (VALIANT) trial. Circulation 2004, 110:1572-1578.

24. Bosch J, Lonn E, Pogue J, Arnold JM, Dagenais GR, Yusuf S: Long-term effects of ramipril on cardiovascular events and on diabetes: results of the HOPE study extension. Circulation 2005, 112:1339-1346.

25. Yusuf S, Gerstein H, Hoogwerf B, Pogue J, Bosch J, Wolffenbuttel BH, Zinman B: Ramipril and the development of diabetes. JAMA 2001, 286:1882-1885.

26. Saxena R, Elbers CC, Guo Y, Peter I, Gaunt TR, Mega JL, Lanktree MB, Tare A, Castillo BA, Li YR, et al: Large-scale gene-centric meta-analysis across 39 studies identifies type 2 diabetes loci. Am J Hum Genet 2012, 90:410-425.

doi:10.1186/1479-5876-11-12

Cite this article as: Karnes et al:: Lack of association of the HMGA1 IVS513insC variant with type 2 diabetes in an ethnically diverse hypertensive case control cohort. Journal of Translational Medicine 2013 11:12.

\section{Submit your next manuscript to BioMed Central and take full advantage of:}

- Convenient online submission

- Thorough peer review

- No space constraints or color figure charges

- Immediate publication on acceptance

- Inclusion in PubMed, CAS, Scopus and Google Scholar

- Research which is freely available for redistribution 